\title{
Human Cells, Tissues, and Cellular and Tissue-Based Products Screening
}

National Cancer Institute

\section{Source}

National Cancer Institute. Human Cells, Tissues, and Cellular and Tissue-Based Products Screening. NCl Thesaurus. Code C133287.

To evaluate and screen Human Cells, T issues, and Cellular and T issue-Based Products (HCT/Ps) donors for communicable disease risks. 